\title{
THE RELATIONSHIP OF THE PROTOPERLARIA AND THE ENDOPTERYGOTA
}

\author{
By PhILlip A. AdAMs \\ Department of Biological Sciences \\ University of California, Santa Barbara
}

The first worker to recognize that the Protoperlaria were a group distinct from the Protorthoptera, and probably ancestral to the Plecoptera, was Tillyard $(1928 \mathrm{a}, \mathrm{b})$. The relationship of these orders has been discussed in more detail by Carpenter (1935). That the Protoperlaria might be of far greater phylogenetic significance has not generally been appreciated. Although the suggestion that the Protoperlaria were close to the ancestral form of the Endopterygota was made by Bradley $(1939,1942)$, this relationship has not previously been documented.

While a comparison was being made between the wings of the protoperlarian, Lemmatophora, and the neuropteran, Sialis, in an effort to determine the venational homologies of the latter, it became apparent that these insects exhibited a number of striking similarities. When the similarity of the wings was noticed, a comparison of other body structures seemed desirable. Since these could not be studied in the fossils, it was necessary to turn instead to the Plecoptera, in the hope that additional resemblances could be found. Such resemblances have been observed, particularly in the sternal region of the thorax, and in the wing articulation; these are discussed briefly below.

The Sialidae are extremely archaic insects; the venation has undergone but little change since the Permian. There are some specializations - fusion of $\mathrm{MP}$ and $\mathrm{CuA}$ in the fore wing, reduction of the anal fan, and lack of nygmatabut in structure and arrangement of the veins the wing remains primitive. No other living insect group shares with the Protoperlaria so many morphological features of 
the wing. Sialis mohri Ross has been used in this study; the other genera of this family differ only slightly. Neohermes californicus (Walker) (Corydalidae) has been used in the study of wing articulation, since the wing base is larger and apparently less specialized than in Sialis.

Material of Lemmatophora typa examined includes the neotype, M. C. Z No. 3536, which is almost perfectly preserved, and upon which Figures 3 and 4 are largely based. Of a long series of impressions of hind wings which were studied, M. C. Z. No. 4425 was best. In none of these fossil specimens were the extreme bases of MP and $\mathrm{CuA}$ preserved.

I should like to acknowledge the kindness of F. M. Carpenter, who has made available the material used in this study, and who, together with W. L. Brown and E. O. Wilson, has contributed many helpful suggestions.

\section{A. Structure of the Veins.}

In Sialis, the veins are not heavily sclerotized, most of them being formed as longitudinal folds in the membrane (Fig. 9b). As a consequence, these veins appear, viewed from one surface, as ridges, and from the other surface, as grooves.

Since the fossil wings are molds of the upper and lower wing surfaces, it has been possible, by viewing under illumination nearly parallel to the surface, to reconstruct a cross-section of the hind wing of Lemmatophora (Fig. 9a). This is similar to that of Sialis in the general structure of the veins, and shares with it a remarkable peculiarity:

\section{Explanation of Plate 13}

Figures 1, 2. Sialis mohri Ross. Anterior and posterior wings, showing venation and distribution of macrotrichia. Figures 3, 4. Lemmatophora typa Sellards. Anterior and posterior wings, showing venation and distribution of macrotrichia. Figure 5. Pteronarcys californica Newport. Portion of anal fan of posterior wing, showing macrotrichia on veins and membrane, and distribution of microtrichia. Figure 6. Sialis mohri. Structure of microtrichia and a macrotrichium. Figure 7. Sialis mohri. Portion of anterior wing, showing distribution of macrotrichia and microtrichia. (Microtrichia indicated by stippling.) Figure 8. Lemmatophora typa. Portion of anterior wing, showing distribution of macrotrichia and microtrichia. (Pigmentation indicated by stippling.) 

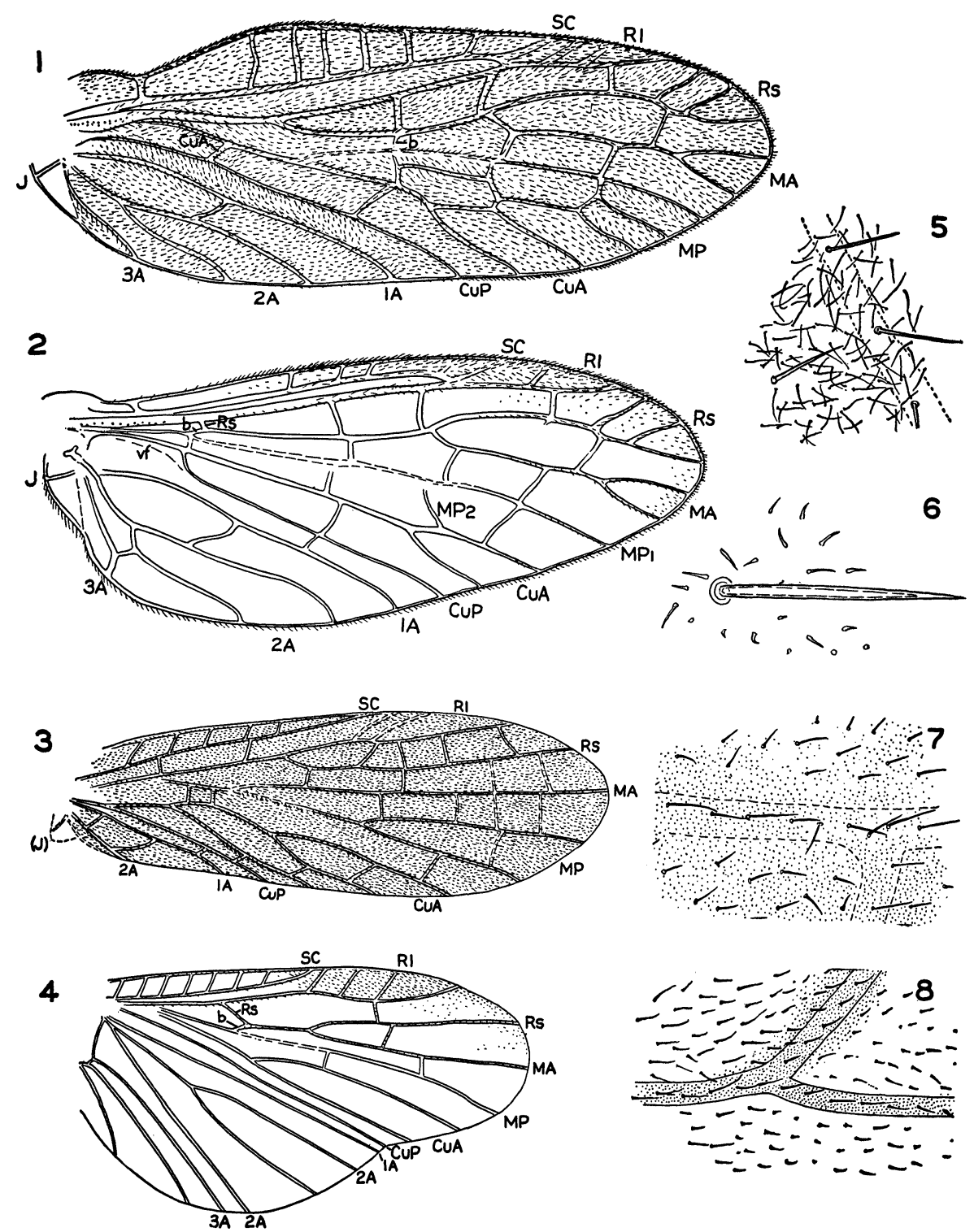

Adams - Protoperlaria and Endopterygota 
$\mathrm{CuA}$ is formed like a trough, appearing as a deep groove when viewed from above, and as a strong ridge, viewed from below. While in Lemmatophora, $\mathrm{CuA}$ is still found in its primitive position, lying on a weak convex fold of the wing, in Sialis this fold has been reversed, so that $\mathrm{CuA}$ lies at the bottom of a concave fold. $\mathrm{CuA}$ in the hind wing of all the other Exopterygota I have examined, with the possible exception of the Caloneurodea, is either indeterminate or on a more or less convex fold as in Lemmatophora. In all the neuropteroids where $\mathrm{CuA}$ is well developed in the hind wing, it is on a concave fold, as in Sialis.

Another peculiarity of the Protoperlaria is the structure of the stem of MP, which frequently is so weak as to be nearly indiscernible. There is little differentiation of the wing membrane along the course of this vein, except for the occurrence of a row of macrotrichia, and its being surrounded by a non-pigmented area. In both wings of Sialis, the stem of MP has a similar structure. This condition is a most unusual one, and its occurrence in Sialis is of considerable significance.

\section{B. Venational Pattern}

The arrangement of veins is fundamentally the same in Sialis and in protoperlarians. In Lemmatophora $\mathrm{RS}_{\mathrm{S}}$ is simple, but is 2- or 3- branched in some other members of this order. $R_{s}$ in sialids also is simple, but for a few marginal veinlets. Fusion of MA with $R_{S}$ is of frequent occurrence among protoperlaria, but is subject to much variation. In Lemmatophora, coalescence of these two veins is clear in the posterior wing, as in the hind wing of Sialis. In the fore wing of Lemmatophora, coalescence does not take place. In Sialis it has, although the connection between MA and the base of MP is not clear as in the hind wing; here a weak crossvein may represent the basal piece of MA, or the basal piece may have moved toward the wing base and disappeared. The basal piece is, however, readily identifiable in the fore wings of most primitive Raphidiodea and Planipennia.

Fusion of $\mathrm{CuA}$ and MP in the fore wing, characteristic of Sialidae and Archisialidae, also occurs in some Proto- 
perlaria (Leucorium, Artinska), but is not ordinarily found among the Planipennia.

Probably the most striking difference between the wings of Lemmatophora and Sialis lies in the shape, there being a well-developed anal fan in the protoperlarian, but none in Sialis. However, a functional fan is still present in the Corydalidae, and a reduced one in the Ithonidae and Polystoechotidae. Anal fans occur elsewhere in the Endopterygota, notably in the Trichoptera and Lepidoptera; one would therefore expect such a structure to be present in the ancestor of the Endopterygota. That its absence in Sialis is due to secondary reduction is indicated by the still relatively broad base of the hind wing. Reduction of the anal fan in Sialis is compatible with a general trend in the Neuroptera toward narrowing of the base of the hind wing.

C. Trichiation of the Wings and Veins

Tillyard (1918) has discussed the trichiation of the wings in the Panorpoid Orders; he distinguishes two types of hairlike structures - macrotrichia, with articulated bases, equivalent to setae, and microtrichia which are simple outgrowths of the cuticle. Macrotrichia are found both on the veins and the wing membrane in many Endopterygota, but among the Exopterygota are largely restricted to the veins. Microtrichia occur in both groups, distributed uniformly over the surface of the wing, including the veins.

Tillyard (1928a) interpreted the fine hairs covering the wing membrane of Protoperlaria as microtrichia; he considered the macrotrichia to be restricted to the veins. This interpretation now appears to be open to question. A careful comparison of these "microtrichia" (Fig. 8) with the macrotrichia of Sialis (Fig. 7) discloses that these structures possess several features in common:

a. Their size is approximately the same.

b. In Lemmatophora, all the setae, whether on the veins or on the membrane, appear to have a definite basal socket, as do those of Sialis. These are distinguishable even in Tillyard's photograph of the wing surface (1928a, Fig. 7). 
c. On the hind wing of both Sialis and Lemmatophora, these hairs are distributed similarly, on the apical part of the costal cell, the stigmatic area, the wing tip, and in rows along the anterior longitudinal

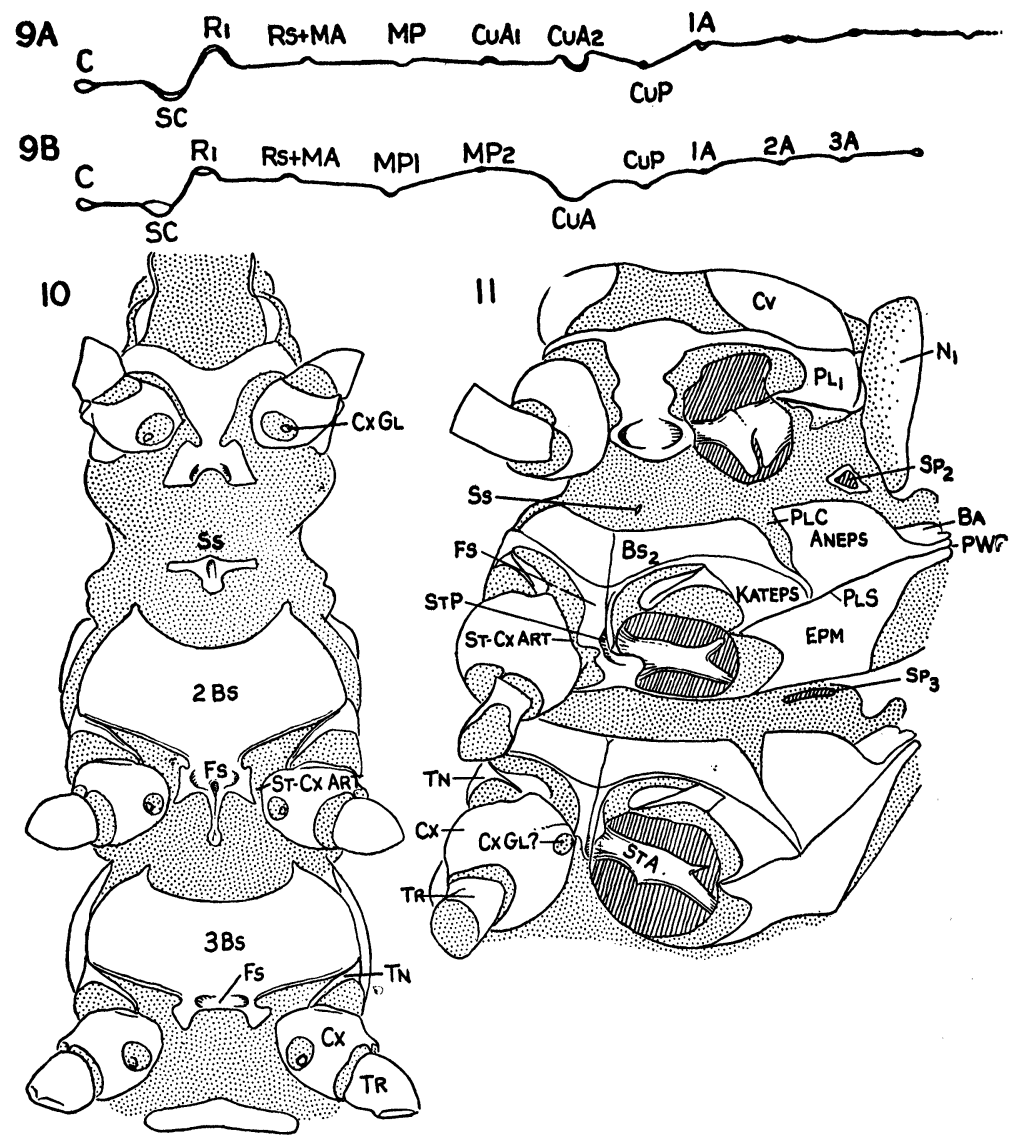

Figures 9-11.

Figure 9A. Lemmatophora typa. Reconstructed cross section of posterior wing. Figure 9B. Sialis mohri. Cross section of posterior wing. Figure 10. Taeniopteryx sp. Ventral view of thorax showing presence of weak sternocoxal articulations. Figure 11. Sialis mohri. Ventrolateral view of thorax, showing sternocoxal articulations, structure of internal skeleton, and general resemblance to Taeniopteryx. 
veins only. There is a decrease in size toward the base of the wing.

In attempting to differentiate between the setae on the veins and those on the membrane, Tillyard greatly overemphasized in his discussion the larger size of the setae on the costa and other veins. While it is true that the costal setae are very slightly larger than those of the membrane, much of the apparent size difference is illusory; in the fossil the setae of the wing membrane, seen against a colorless background, look brown, while those on the veins, seen against a brown background, look black, and therefore heavier.

Even though some size difference exists, this is not in itself sufficient reason for interpreting the hairs on the veins as macrotrichia, and those on the membrane as microtrichia, for in Sialis there is a similar size difference.

Furthermore, the microtrichia of Sialis (indicated by stippling in Figure 7, and shown in more detail in Figure 6) are very small, about the same size as the grain of the matrix in which are imbedded the fossils. Even were similar microtrichia present in Lemmatophora, as was almost certainly the case, they would therefore be obscured by the grain of the rock.

Tillyard's reluctance to recognize the macrotrichia on the wing membrane of Lemmatophora as such was probably due to an impression that macrotrichia do not occur in this location among the Exopterygota. While this is generally true, there is at least one exception. Since the Plecoptera are considered to be descended from the Protoperlaria, an examination was made of the wing surface of a representative of this order, Pteronarcys californica Newport. In this insect, prominent microtrichia are scattered over the entire wing. Small macrotrichia are sparsely distributed on the veins of the fore wing. But on the anal fan of the hind wing near the margin, large macrotrichia occur not only on the veins, but also on the wing membrane (Fig. 5).

From the above evidence, it seems reasonable to conclude that macrotrichia were present on both the veins and the 
wing membrane of the Protoperlaria, in size and distribution almost identical to those of Sialis.

D. The Thorax.

The sternum of the endopterygote thorax differs from that of the primitive Exopterygota in having a reduced furcasternum. The coxae of the Endopterygota have acquired a third point of articulation, on the furcasternum.

In the Plecoptera, the sternum is highly variable. Most studies of the thorax in this order have been made of Pteronarcys, or the large perlidae. In these stoneflies, the sternum is large and broad, and has slight resemblance to that of Sialis. But in some of the smaller stoneflies, such as Taeniopteryx, this is not the case.

The furcasternum (Fs) of Taeniopteryx (Fig. 10) is small, about the size as is that of Sialis. On both the mesothorax and metathorax, it bears on each side a process which extends close to the coxa; these have been termed furcasternal arms by Hanson (1946), who states "Although they are not articulated with the coxae, they appear to be adapted to offer them very strong support in the movement of the legs." The sternal coxal articulation (ST-CX ART) of the neuropteroid thorax is probably its most distinctive feature; one would expect this condition to have been derived from exactly such a stage as occurs in some stoneflies, by gradual strengthening of the association of the coxa and furcasternum. Once a sternal coxal articulation had become established, the thorax could be greatly strengthened by an infolding along the midline, reducing the exposed area of the furcasternum, so that the coxal bases become nearly contiguous. That such an infolding has taken place is indicated, not only by the structure of the furcasternum of neuropteroids, but also by the median longitudinal sutures on the basisterna (Fig. 11).

It is not necessarily to be inferred from the close resemblance of the thorax of Taeniopteryx to that of Sialis, that taeniopterygids are more primitive or more closely related to the Endopterygota than are other stoneflies. It is apparent, though, that these thoraces are constructed on the same general plan, and show remarkably similar capabilities for structural modification. 
It is interesting to note, while reference is being made to the figure of the thoracic sterna of Taeniopteryx, the occurrence of openings of coxal glands (CxGl) on membranous areas of each coxa. Similar membranous areas occur on the hind coxae of Sialis, Neohermes, Corydalus, and Chauliodes, but in none of these insects has it been possi-
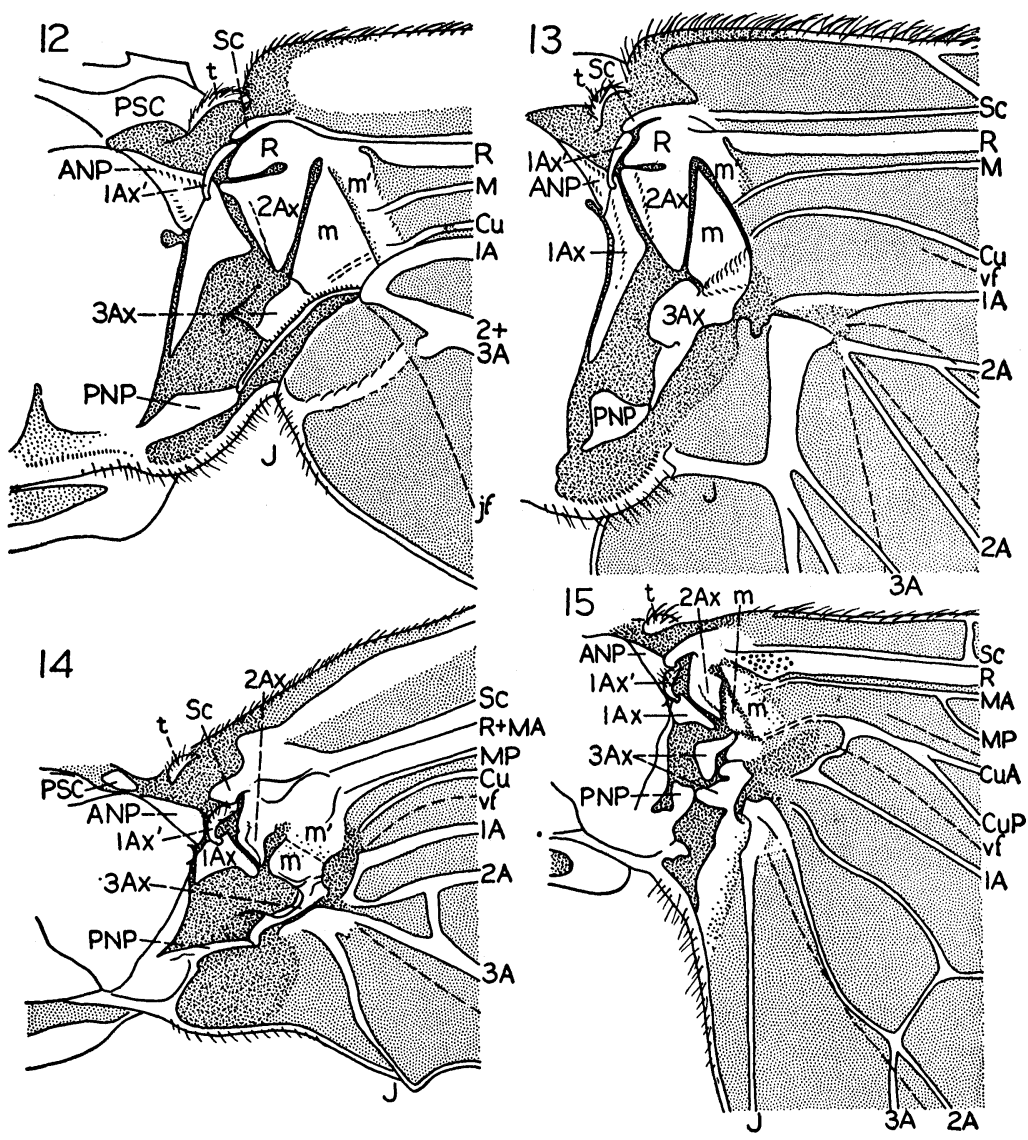

Figures 12-15.

Figure 12. Pteronarcys californica (Plecoptera). Base of right anterior wing. Figure 13. P. californica. Base of posterior wing. Figure 14. Neohermes californicus (Walk.) (Megaloptera). Base of anterior wing. Figure 15. N. californicus. Base of posterior wing. 
ble to demonstrate by gross dissection any associated glandular structure. There is frequently, however, a small papilliform projection from one coxa interlocking with the membranous area of the other, apparently serving to restrict movement of the hind coxae.

E. Wing Articulation.

The axillary sclerites of Plecoptera (Pteronarcys, Figs. 12-13) and Megaloptera (Neohermes, Figs. 14-15) resemble each other in relatively large size, simple shape, and light degree of sclerotization. The wing base of Pteronarcys is the more generalized, while that of Neohermes appears to be modified for greater strength and flexibility. Only a few simple changes would be necessary to derive the more specialized wing base of Neohermes from that of Pteronarcys.

In Neohermes, the long posterior arm of the first axillary has been lost, and the anterior arm, which is connected to the basal process of Sc, is strengthened. The head of $R$ has disappeared; this structure appears to be non-functional in Pteronarcys, and the articulation stronger without it. The third axillaries of the anterior wing are similar, but there has been a shift in the axis of articulation of the median plate with the second axillary, in Neohermes. In the anterior wing of both Neohermes and Pteronarcys, the third axillary articulates on a long, slender posterior notal wing process. In the posterior wing, however, the structures of third axillary and posterior notal wing process differ greatly. In Neohermes the third axillary is dissected, the median plate, flexor muscle attachment, and posterior articulating portion being separate. This condition, however, is not typical of all Neuroptera. It is also most unusual for the posterior wing process to remain attached to the metanotum; in nearly all other Neuroptera it is a separate sclerite, as in Pteronarcys.

The difference in articulation of the posterior wing may be due in large part to suppression of the anal fan in the Neuroptera. In Neohermes, although a functional fan is still present, the number of veins is small, and this region of the wing bears little resemblance to that of Pteronarcys. 


\section{F. CONCLUSIONS.}

Some evidence for a close relationship between the Protoperlaria and the Neuroptera has been discussed above. This evidence, summarized below, consists in part of distinctive wing characters which are shared by the archaic Sialidae and the Protoperlaria (a), and in part of some similarities between the Plecoptera (derivatives of the Protoperlaria) and the Neuroptera (b). To these may be added some general characters which would of necessity be expressed in any group ancestral to the Endopterygota (c).

a. Distinctive common features of the protoperlarian and sialid wing are:

1. The density and arrangement of veins and crossveins is about the same; in particular, Rs has usually only one or two branches.

2. There is a strong tendency for coalescence of MA and Rs.

3. The stem of MP is weak.

4. $\mathrm{CuA}$ of the hind wing of Protoperlaria is structurally similar to that of Sialis, the upper surface being deeply grooved.

5. The distribution of macrotrichia on the membrane and veins is about the same.

b. Features shared by the Plecoptera and Neuroptera, indicative of relationship are:

6. Furcasternum with a coxal articulation.

7. Generally similar shape and arrangement of axillary sclerites.

c. Other features of the Protoperlaria which are not so distinctive, but which are consistent with their probable role as ancestors of the Endopterygota are:

8. A well-developed anal fan.

9. Cerci

10. Ovipositor.

11. Tibial spurs, present not only at the apex, but in a series along the length of the tibia.

12. Five-segmented tarsi. 
In addition to the above morphological evidence, a close relationship of the Protoperlaria and the Neuroptera is in agreement with recent theoretical work on the origin of the larva, and the significance of the pupal stage. The nymphs of the Protoperlaria were described in detail by Carpenter (1935); they appear to have been similar to those of the Plecoptera. Presumably, there was a large number of nymphal instars, as is the case in the Plecoptera, some species of which have more than thirty.

Bradley (1942) recognized the significance of the Protoperlaria, suggesting them as possible ancestors of the Endopterygota because they are the most generalized known Neoptera, and because of some similarities in development of their close relatives, the Plecoptera, and of Sia $i$ s. More recently. Sharov $(1953,1957)$ has developed a theory of the origin of holometabolous development from an insect with a large number of immature stadia, including both larval and nymphal stages, and with several imaginal instars. Of the living Neoptera, the Plecoptera, with their large number of immature stages, approach closest to this condition.

The evidence described above appears sufficient to justify the statement that a close relationship probably exists between the Protoperlaria and the Neuroptera; moreover. there is no known feature of the Protoperlaria which would prevent their being considered directly ancestral to the Endopterygota.

Bradley, J. C.

\section{Literature Cited}

1939. A laboratory guide to the study of the evolution of the wings of insects. Daw, Illston, Ithaca, N. Y. 60 pp., 83 pl.

1942. The origin and significance of metamorphosis and wings among insects. Proc. 8th Amer. Sci. Cong. 3:303-309.

Carpenter, F. M.

1935. The Lower Permian insects of Kansas. Part 7. The order Protoperlaria. Proc. Amer. Acad. Art Sci. 70:103-146.

Hanson, J. F.

1946. Comparative morphology and taxonomy of the Capniidae (Plecoptera). Amer. Mid. Nat. 35.193-249 
Sharov, A. G.

1953. Development of the Thysanura in connection with the problem of the phylogeny of insects. Trudy Inst. Animal Morphol. USSR 8:63-127. (In Russian.)

1957. Types of insect metamorphosis and their interrelationships. Ent. Obozrenie 36:569-576. (In Russian.)

Tillyard, R. J.

1918-1919. The panorpoid complex. Proc. Linn Soc. N. S. W. 43:265284, 286-319, 626-657; $44: 533-718$.

1928a. Kansas Permian Insects. Part 10. The new order Protoperlaria: a study of the typical genus Lemmatophora Sellards. Amer. J. Sci. $16: 185-220$.

1928b. Kansas Permian Insects. Part II. Order Protoperlaria: Family Lemmatophoridae (Continued). Amer. J. Sci. 16:313-348.

\section{Lettering Used in Figures}

1A, 2A, 3A - first, second, and third anal veins.

ANEPS - anepisternum

ANP - anterior notal wing process

$1 \mathrm{Ax}^{\prime}$ - anterior arm of first axillary sclerite

b - basal piece of media anterior

BA - basalare

C - costa

$\mathrm{CuA}$ - cubitus anterior

$\mathrm{CuP}$ - cubitus posterior

$\mathrm{Cx}$ - coxa

CxGl - membranous area bear-

ing opening of coxal gland

EPM - epimeron

EPS - episternum

Fs - furcasternum

$\mathbf{J}$ - jugal vein

jf - jugal fold

KATEPS - katepisternum

m.m' - medial plates
MA - media anterior

MP - media posterior

$\mathrm{N} 1$ - pronotum

PLC - pleural cleft

PLS - pleural suture

PNP - posterior notal wing process

PSC - prescutum

PWP - posterior notal wing process

$\mathrm{R} 1$ - anterior branch of radius

$\mathrm{Rs}$ - radial sector

SA - subalare

SC - subcosta

SP - spiracle

Ss - spinasternum

STA - sternal apophysis

ST-CX ART - sterno-coxal articulation

t - tegula

$\mathrm{TN}$ - trochantin

$\mathrm{TR}$ - trochanter

vf - vannal fold 

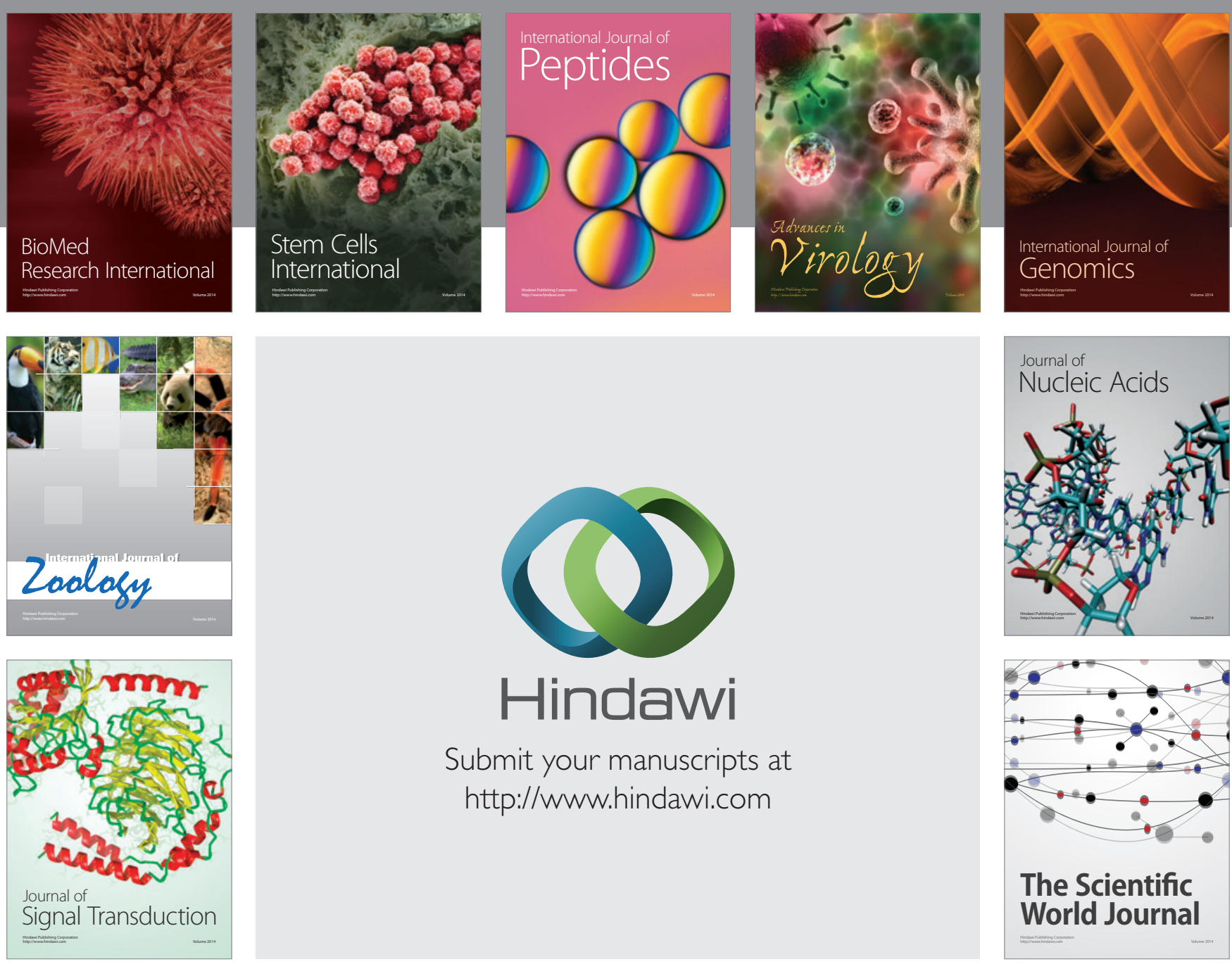

Submit your manuscripts at

http://www.hindawi.com
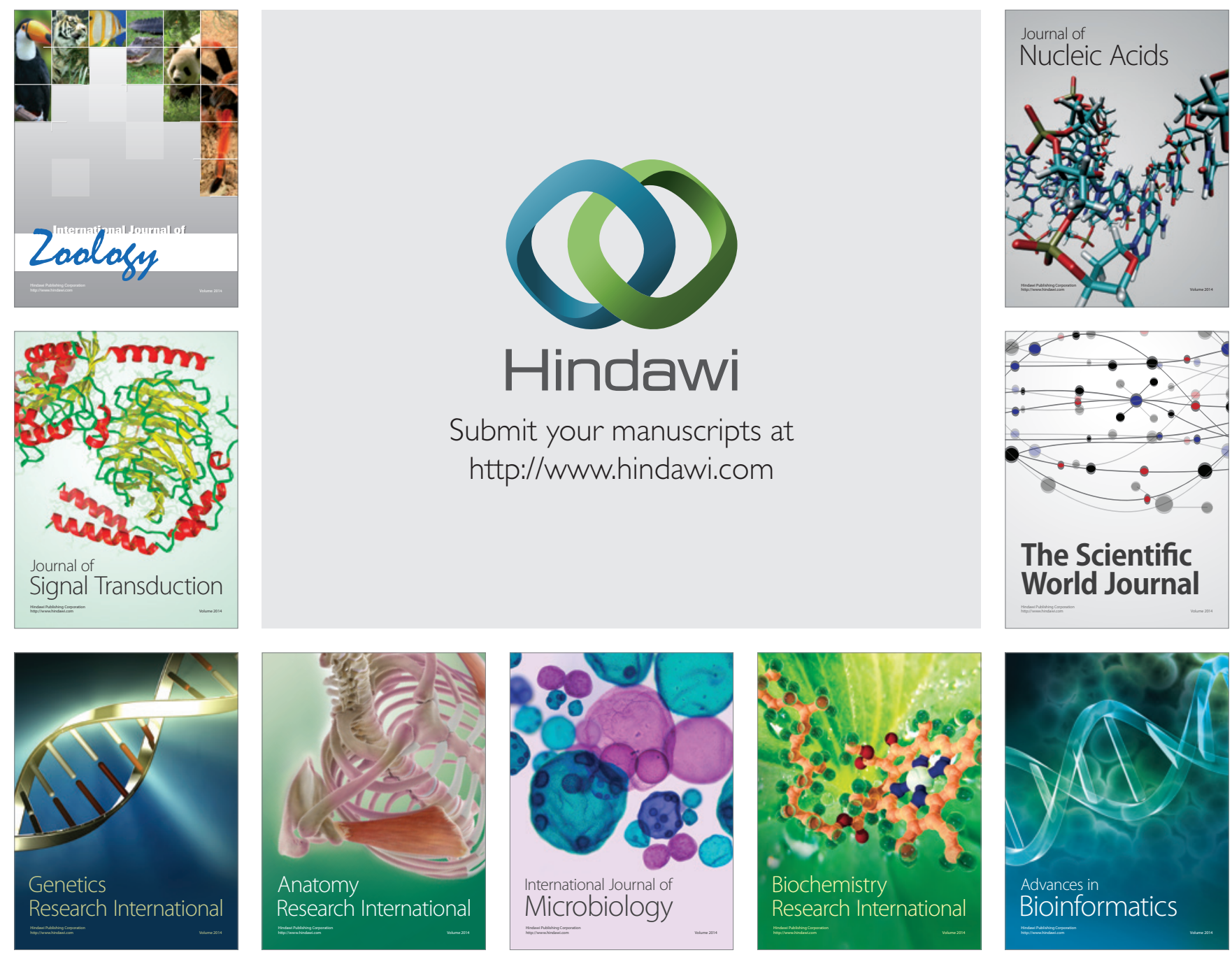

The Scientific World Journal
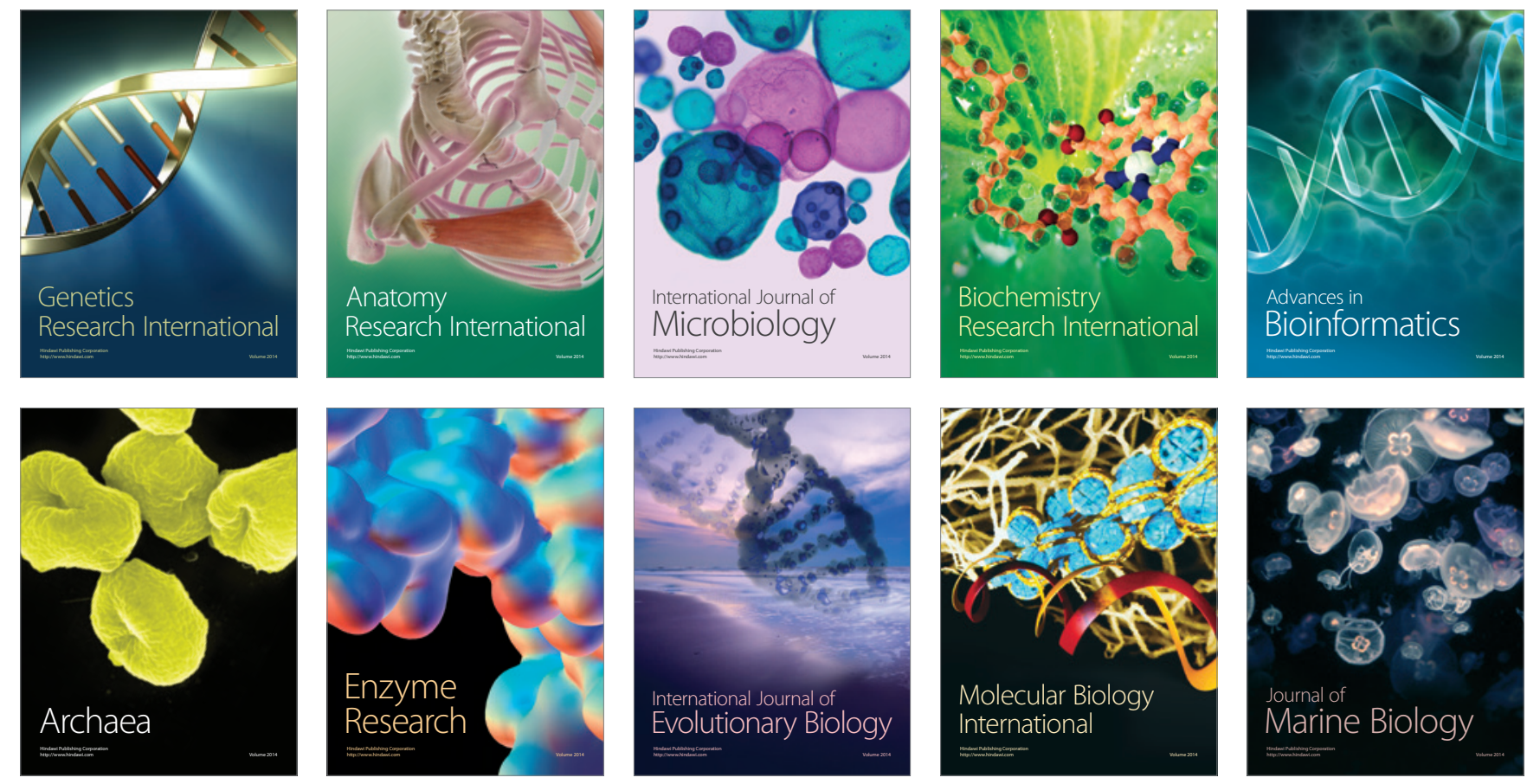